\title{
THE RIGIDITY PROBLEM FOR STABLE SPACES
}

\author{
DONALD W. KAHN
}

\begin{abstract}
This note treats the problem of when the group of homotopy self-equivalences of a space is trivial. For stable spaces, with finitely many nonvanishing homotopy groups, we give a complete solution in an inductive sense. One of the consequences of this result is that for any stable space, with precisely two nonvanishing homotopy groups, the group of self-equivalences is nontrivial.
\end{abstract}

If $X$ is a (pointed) path connected space, we denote by $G(X)$ the group of homotopy classes of homotopy equivalences from $X$ to itself (all preserving base point). This is the homotopy theorist's analogue of the group of automorphisms of a group, and it has now been studied by many authors. For example, C. Wilkerson [8] and D. Sullivan [7] have shown that when $X$ is a simply-connected finite complex, $G(X)$ is a finitely-presented group. Their methods, refining methods in [4], use algebraic groups, and they are not fine enough to get closer to $G(X)$ than a subgroup of finite index, or a quotient by a finite, normal subgroup. On the other hand, there is a space $X$, which at every prime but 3 is a $K(Z, 4)$, but for which $G(X)$ is trivial [5]. Since $G(K(Z, 4))=Z_{2}$, the integers mod 2 , we see that a space $X$ may be equivalent to a space $Y$, at all but one prime, yet $G(X)$ is trivial and $G(Y)$ is not. We shall call a space $X$, for which $G(X)$ is trivial, rigid, and we propose to study such spaces-in a stable sense-in this paper.

We shall work in a category whose objects are spaces with finitely-many nonvanishing homotopy groups, in a stable range. Specifically, such a space $X$ shall be endowed with a finite Postnikov tower

$$
X=X_{k} \stackrel{\pi_{k}}{\rightarrow} \cdots \stackrel{\pi_{n+1}}{\rightarrow} X_{n}
$$

where $\pi_{i}$ is a principal fibration with fibre $K\left(\pi_{i}(X), i\right), n>1$, and $k<2 n-$ 1. Such a space is infinitely de-loopable, and our continuous maps and homotopies shall be presumed to be infinitely de-loopable also. Base points are assumed but omitted from the notation. We denote this category by $\delta_{1}$. For comparison purposes-at the end of this note-we denote by $\mathcal{S}_{2}$ the category of simply-connected finite complexes, and stable (in the sense of suspension) homotopy classes of maps.

In a given Postnikov system, if $j>i$, there is a natural homomorphism (see [2])

$$
\phi_{j, i}: G\left(X_{j}\right) \rightarrow G\left(X_{i}\right),
$$

Received by the editors June 29, 1978 and, in revised form, September 5, 1978.

AMS (MOS) subject classifications (1970). Primary 55D10; Secondary 55D35, 55D40.

Key words and phrases. Homotopy equivalences, stable spaces, Postnikov tower. 
which is studied in [3]. The image of $\phi_{m+1, m}$ is precisely known, while the kernel is there related to certain cohomology groups and extensions.

Our idea is now to study the rigidity problem, that is when a space is rigid, by induction. We easily characterize when $K(\pi, n), n>1$, is rigid (Proposition 2), and then go on to give necessary and sufficient conditions for $\operatorname{ker}\left(\phi_{m+1, m}\right)$ to be trivial. As a corollary, we may conclude that there is no space $X$ in $\delta_{1}$, with precisely two nonvanishing homotopy groups, which is rigid. In conclusion, we compare this situation with that in the category $\delta_{2}$. We recall (Proposition 3) the well-known result that there is no nontrivial $X$ in $\delta_{2}$, which is rigid. We then show (Proposition 4) that if $X_{k}$ is a Postnikov term for a finite stable complex $X$, and $X_{k}$ is rigid, then $\operatorname{dim}(X) \geqslant k$.

Finally, we would like to point out that our results for $\mathcal{S}_{1}$ do not require the homotopy groups of the spaces in question to be finitely-generated, as opposed to many of the other papers in the field. For example, our Proposition 2 actually holds for all Abelian groups $\pi$, if we choose $X$ to be cellular.

Proposition 1. If $X$ is a rigid space, in the category $\varsigma_{1}$, then the identity map $1_{X}$ has order 2 in the sense of loop multiplication in $X$. In particular, every element in the homotopy or stable homology groups of $X$ has order 2.

Proof. Let $[X, X]$ denote the Abelian group of homotopy classes of maps from $X$ to itself, under loop multiplication. Assume that the order of the class of $1_{X}$ is different from 2 . Then $-1_{X}$ is not homotopic to $1_{X}$. As $-1_{X}$ is clearly a homotopy equivalence, $G(X)$ has at least two elements.

For the rest of the proposition, we simply observe that the addition in $[X, X]$ acts additively on homotopy and stable homology (that is homology groups in the stable range).

Proposition 2. Let $X$ be a space in $\widehat{S}_{1}$, with a single, nonvanishing homotopy group, i.e., $X=K(\pi, n), n>1$. Then $X$ is rigid precisely when $\pi=Z_{2}$, the integers mod 2.

Proof. If $X$ is rigid, then we know that every element of $\pi$ has order 2. I claim that $\pi$ is a $Z_{2}$-vector space. It is trivial to construct a group homomorphism from a $Z_{2}$-vector space $V$ onto $\pi$, say $g: V \rightarrow \pi$. But the kernel of $g$ is trivially checked to be a vector subspace, proving that $\pi$ is the quotient of a vector space by a subspace, and hence, $\pi$ is itself a vector space.

Now, it is well known that $G(K(\pi, n))=\operatorname{Aut}(\pi)$, the group of automorphisms of $\pi$. But the only $Z_{2}$-vector space, without a nontrivial automorphism, has dimension 1.

Before stating our main theorem, it will be convenient to specify some notation.

a. $\Sigma X$ means the (reduced) suspension of $X$. $\Sigma f$ means the suspension of the map $f . \sigma: H^{p}(Y) \stackrel{\approx}{\rightarrow} H^{p+1}(\Sigma Y)$ is the usual suspension isomorphism in cohomology. $\Omega X$ means loops on $X$. 
b. If $Y$ is $(n-1)$-connected, we shall freely write $i_{n} \in H^{n}\left(Y ; \pi_{n}(Y)\right)$ for the fundamental class. If $x \in H^{m}(Y ; \pi)$, write $\phi_{x}: Y \rightarrow K(\pi, m)$ for a map such that $\phi_{x}^{*}\left(i_{m}\right)=x$.

c. For a fibration in a given Postnikov tower, say

$$
X_{n+1} \stackrel{\pi_{n+1}}{\rightarrow} X_{n},
$$

we shall abbreviate the fibre $K\left(\pi_{n+1}(X), n+1\right)$ to $K$. We write the inclusion of the fibre $i: K \rightarrow X_{n+1}$.

THEOREM. Let $X$ be a space in $\varsigma_{1}$, and let

$$
\phi_{m+1, m}: G\left(X_{m+1}\right) \rightarrow G\left(X_{m}\right)
$$

be the homomorphism described above. Then a necessary and sufficient condition that $\operatorname{ker}\left(\phi_{m+1, m}\right)$ be trivial, is that for every cohomology class

$$
x \in H^{m+1}\left(X_{m+1} ; \pi_{m+1}(X)\right),
$$

for which $i \cdot \phi_{x}+1_{X_{m+1}}$ induces an isomorphism on homology in dimension $m+1$, there is a map

$$
\alpha: \Sigma X_{m+1} \rightarrow X_{m}
$$

so that $\alpha^{*}\left(k^{m+2}\right)=\sigma(x)$, with $k^{m+2}$ being the $k$-invariant of the fibration.

Proof. We are working in the stable category $S_{1}$. If we suppose $\{f\} \in$ $\operatorname{ker}\left(\phi_{m+1, m}\right)$, then the diagram

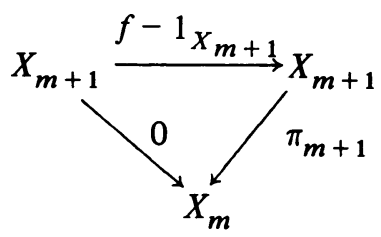

is homotopy commutative ( 0 sends all $X_{m+1}$ to the base point).

By exactness, there is a map $\psi_{f}: X_{m+1} \rightarrow K=\pi_{m+1}^{-1}$ (pt.), so that

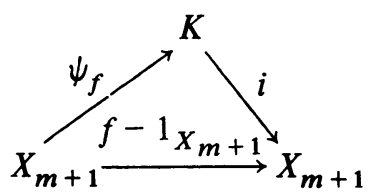

is homotopy commutative.

Now, the fibration $\pi_{m+1}$ gives rise to an exact sequence of spaces

$$
\cdots \rightarrow \Omega X_{m} \stackrel{\gamma}{\rightarrow} K \stackrel{i}{\rightarrow} X_{m+1} \stackrel{n_{m+1}}{\rightarrow} X_{m} \rightarrow \cdots ;
$$

it is well known that if we identify cohomology of $\Sigma \Omega X_{m}$ and $X_{m}$ in the stable range, then $\sigma\left(\gamma^{*}\left(i_{m+1}\right)\right)=k^{m+2}$, the $k$-invariant.

By exactness, the map $f-1_{X_{m+1}} \simeq i \cdot \psi_{f}$ will be null-homotopic (and hence $f \simeq 1_{X_{m+1}}$ ) precisely when there is a map $\alpha^{\prime}: X_{m+1} \rightarrow \Omega X_{m}$, with 


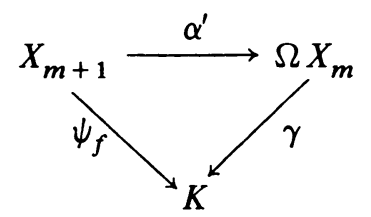

being homotopy commutative.

If we suspend this diagram and write $\alpha=\Sigma \alpha^{\prime}$, then stabily we get a homotopy commutative diagram

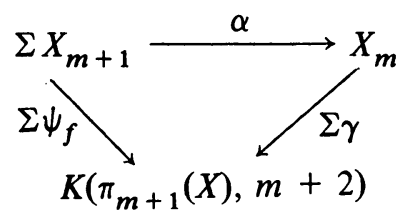

On cohomology, $\alpha^{*}\left(k^{m+2}\right)=\left(\Sigma \psi_{f}\right)^{*}\left(i_{m+2}\right)=\sigma\left(\psi_{f}^{*}\left(i_{m+1}\right)\right)$.

To complete the proof, we must see that the classes $x$, with the condition given in the statement of the theorem, are precisely those classes of the form $\psi_{f}^{*}\left(i_{m+1}\right)$. But given such a class $x, i \cdot \phi_{x}+1_{X_{m+1}}$ clearly induces isomorphisms on homology in dimensions through $m+2$ (recall $H_{m+2}(K)=0$ ) and is thus a homotopy equivalence $f$. Then $f-1_{X_{m+1}} \simeq i \cdot \phi_{x}$, so we take $\phi_{x}=\psi_{f}$ and $\psi_{f}^{*}\left(i_{m+1}\right)=\phi_{x}^{*}\left(i_{m+1}\right)=x$. On the other hand, given $\psi_{f}$ for a homotopy equivalence $f$, with $\{f\} \in \operatorname{ker}\left(\phi_{m+1, m}\right)$-the class $\psi_{f}^{*}\left(i_{m+1}\right)$ clearly meets the condition of the theorem.

We remark that the theorem is clearly valid for $k\left(\phi_{j, i}\right), j>i$, when the homotopy groups in dimensions between $i$ and $j$ vanish.

COROLlaRY. If $X$ is a space in $\mathcal{S}_{1}$ with precisely two nonvanishing homotopy groups, $X$ is not rigid.

Proof. By our propositions, we assume the two nonvanishing groups $\pi_{i}(x)$ and $\pi_{j}(x), i<j<2 i-1$, are both direct sums of copies of $Z_{2}$. We shall prove $\operatorname{ker}\left(\phi_{j, i}\right)$ is nontrivial.

By [6], the $Z_{2}$-cohomology of $K\left(Z_{2}, i\right)$ is nonzero in every dimension not less than $i$. In particular, the subgroup

$$
H^{j}\left(K\left(\pi_{i}(X), i\right) ; \pi_{j}(X)\right) \subseteq H^{j}\left(X_{j} ; \pi_{j}(X)\right)
$$

is nontrivial. Let $x \neq 0$ be a class in this subgroup. Consider the map

$$
X_{j}=X \stackrel{\phi_{x} \times 1_{X}}{\rightarrow} K\left(\pi_{j}(X), j\right) \times X \stackrel{\bar{\mu}}{\rightarrow} X,
$$

where $\bar{\mu}$ is the action of the fibre. It clearly induces isomorphisms on homotopy groups and is thus a homotopy equivalence. Stably, it is just $i \cdot \phi_{x}+1_{x}$.

We must ask whether there is $\alpha: \Sigma X \rightarrow X_{i}$ with $\alpha^{*}\left(k^{j+1}\right)=\sigma(x) \neq 0$. But 
$\Sigma X$ is $i$-connected and $X_{i}=K\left(\pi_{i}(X), i\right)$, so every $\alpha$ is null-homotopic.

Remarks. 1. Combining [3] with this Corollary, it is not hard to show that if $X$ is in $\delta_{1}$ and every $k$-invariant is obtained from the fundamental class by a primary cohomology operation, then $X$ is not rigid.

2. It would be interesting to get information on $G(X)$ for spaces $X$ with 3 or more homotopy groups. If $X$ is rigid, then there is some $j$ with $G\left(X_{j}\right)$ nontrivial, yet $G\left(X_{j+1}\right)$ is trivial.

We now compare these results with the category of finite complexes $\delta_{2}$. The following is well known.

Proposition 3. If $X$ is a nontrivial, finite, path connected complex, the group of stable self-equivalences is nontrivial. (That $X$ is nontrivial means $X$ does not have the homotopy-type of a point.)

Proof. D. Anderson and M. Barratt have proved (unpublished) that the additive stable order of $1_{X}$ cannot be 2 . (This may be shown by looking at the Steenrod operation $S q^{1}$ in $X \wedge X$. If the stable order of $1_{X}$ is 2 , the same would be true for $1_{X \wedge X}$. Taking $u \in H^{i}\left(X: Z_{2}\right)$, with $S q^{1} u \neq 0$, of maximal dimension, we get $S q^{2}(u \wedge u) \neq 0$, which quickly leads to a contradiction. Or one may study top dimensional cells of $X$, and copy the proof (as in [1]) that the homotopy groups of $S^{n} \cup_{2} e^{n+1}$ have an element of order 4).

Then just as before, $1_{X}$ and $-1_{X}$ represent different elements in our group.

Proposition 4. Let $Y$ be a connected, finite complex $(i-1)$-connected, $i>1$, and $\operatorname{dim} Y<2 i-1$. Let $Y_{j}$ be a Postnikov term for $Y, i \leqslant j<2 i-1$. If $Y_{j}$ is rigid, then $j \leqslant \operatorname{dim}(Y)$.

Proof. By Proposition 3, it suffices to show that if $j>\operatorname{dim}(Y)+1$, $G(Y)=G\left(Y_{j}\right)$.

Clearly, any two homotopy equivalences, which represent the same class on $Y_{j}$, must be homotopic, for $Y$ and $Y_{j}$ have the same $j$-type.

On the other hand, the fibration $p: Y \rightarrow Y_{j}$ has a section over the $(j+1)$ skeleton $S: Y_{j}^{(j+1)} \rightarrow Y$. If $f: Y_{j} \rightarrow Y_{j}$ is a (cellular) homotopy equivalence, we may follow $Y \stackrel{p}{\rightarrow} Y_{j} \stackrel{f}{\rightarrow} Y_{j}$ by $S$, to get a homotopy equivalence of $Y$ to itself, which maps to the class of $f$. Thus the natural map $G(Y) \rightarrow G\left(Y_{j}\right)$ is also onto.

REMARK. On the negative side of things, this paper shows that nonrigidity is almost everywhere in stable homotopy, and the consequences-such as a plurality of possible $k$-invariants for a space-cannot be avoided.

\section{REFERENCES}

1. P. Hilton, Introduction to homotopy theory, Cambridge Univ. Press, Cambridge, 1953.

2. D. W. Kahn, Induced maps for Postnikov systems, Trans. Amer. Math. Soc. 107 (1963), 432-450.

3. The group of homotopy equivalences, Math. Z. 84 (1964), 1-8.

4. ___, The group of stable self-equivalences, Topology 11 (1972), 133-140. 
5. Realization problems for the group of homotopy classes of self-equivalences, Math. Ann. 2220 (1976), 37-46.

6. J.-P. Serre, Cohomologie Mod 2 des complexes de Eilenberg-Mac Lane, Comment. Math. Helv. 27 (1953), 198-232.

7. D. Sullivan, Infinitesimal computations in topology, Inst. Hautes Ėtudes Sci. Publ. Math. 47 (1977), 269-332.

8. C. Wilkerson, Applications of minimal simplicial groups, Topology 15 (1976), 111-130.

School of Mathematics, University of Minnesota, Minneapolis, Minnesota 55455 